This item was submitted to Loughborough's Research Repository by the author.

Items in Figshare are protected by copyright, with all rights reserved, unless otherwise indicated.

\title{
Dynamical density functional theory and its application to spinodal decomposition
}

PLEASE CITE THE PUBLISHED VERSION

http://dx.doi.org/10.1063/1.1778374

PUBLISHER

(C) American Institute of Physics

VERSION

VoR (Version of Record)

LICENCE

CC BY-NC-ND 4.0

REPOSITORY RECORD

Archer, Andrew J., and Robert Evans. 2019. "Dynamical Density Functional Theory and Its Application to Spinodal Decomposition”. figshare. https://hdl.handle.net/2134/15985. 


\section{A $\mid$ The Journal of Chemical Physics}

Dynamical density functional theory and its application to spinodal decomposition

A. J. Archer and R. Evans

Citation: The Journal of Chemical Physics 121, 4246 (2004); doi: 10.1063/1.1778374

View online: http://dx.doi.org/10.1063/1.1778374

View Table of Contents: http://scitation.aip.org/content/aip/journal/jcp/121/9?ver=pdfcov

Published by the AIP Publishing

Articles you may be interested in

Dynamic density functional theory with hydrodynamic interactions and fluctuations

J. Chem. Phys. 140, 234115 (2014); 10.1063/1.4883520

Extended dynamical density functional theory for colloidal mixtures with temperature gradients

J. Chem. Phys. 137, 224904 (2012); 10.1063/1.4769101

Spinodal decomposition of a confined colloid-polymer system

J. Chem. Phys. 137, 204902 (2012); 10.1063/1.4767399

Derivation of dynamical density functional theory using the projection operator technique

J. Chem. Phys. 131, 244101 (2009); 10.1063/1.3266943

Dynamic density functional theory for steady currents: Application to colloidal particles in narrow channels

J. Chem. Phys. 119, 1766 (2003); 10.1063/1.1582434

\section{AIP $\mid$ Chaos \\ CALL FOR APPLICANTS \\ Seeking new Editor-in-Chief}




\title{
Dynamical density functional theory and its application to spinodal decomposition
}

\author{
A. J. Archer ${ }^{\mathrm{a})}$ and R. Evans \\ H.H. Wills Physics Laboratory, University of Bristol, Bristol BS8 1TL, United Kingdom
}

(Received 5 May 2004; accepted 9 June 2004)

\begin{abstract}
We present an alternative derivation of the dynamical density functional theory for the one-body density profile of a classical fluid developed by Marconi and Tarazona [J. Chem. Phys. 110, 8032 (1999)]. Our derivation elucidates further some of the physical assumptions inherent in the theory and shows that it is not restricted to fluids composed of particles interacting solely via pair potentials; rather it applies to general, multibody interactions. The starting point for our derivation is the Smoluchowski equation and the theory is therefore one for Brownian particles and as such is applicable to colloidal fluids. In the second part of this paper we use the dynamical density functional theory to derive a theory for spinodal decomposition that is applicable at both early and intermediate times. For early stages of spinodal decomposition our nonlinear theory is equivalent to the (generalized) linear Cahn-Hilliard theory, but for later times it incorporates coupling between different Fourier components of the density fluctuations (modes) and therefore goes beyond CahnHilliard theory. We describe the results of calculations for a model (Yukawa) fluid which show that the coupling leads to the growth of a second maximum in the density fluctuations, at a wave number larger than that of the main peak. (C) 2004 American Institute of Physics.
\end{abstract}

[DOI: $10.1063 / 1.1778374]$

\section{INTRODUCTION}

Classical density functional theory $(\mathrm{DFT})^{1}$ is a remarkably successful theory for describing the rich behavior of the equilibrium structure and thermodynamics of fluids in external potentials. DFT has been used to describe a wide variety of fluid interfacial, confinement and even freezing phenomena; for example, DFT has been a vital tool in understanding the wetting behavior and surface phase transitions of fluids adsorbed on various substrates. ${ }^{1,2}$ Given the success of DFT for describing static inhomogeneous fluids, it is of great interest to be able to build upon and incorporate these theories into a theory for the dynamics of inhomogeneous fluids.

There have been several approaches to obtaining an equation of motion for the one-body density profile $\rho(\mathbf{r}, t)$ of a classical fluid. The form that these theories takes depends somewhat on how the particular authors define $\rho(\mathbf{r}, t)$. For some, $\rho(\mathbf{r}, t)$ is an "ensemble" average over the possible configurations of the system at time $t$, given an ensemble of starting configurations at an earlier time $t=0$. Using this definition for $\rho(\mathbf{r}, t)$, there is clearly a unique density profile $\rho(\mathbf{r}, t)$ at a given time $t$, and therefore the equation governing the dynamics of this density profile will be deterministic. This is the philosophy behind the approach of Marconi and Tarazona (MT) in Refs. 3 and 4. Their approach obtains more formally some of the results proposed by earlier authors, such as Evans ${ }^{5}$ and Dieterich et al., ${ }^{6}$ where it is assumed from the outset that the gradient of the chemical potential $\nabla \mu(\mathbf{r}, t)$ is the thermodynamic force driving a particle current

${ }^{a)}$ Electronic mail: andrew.archer@bristol.ac.uk

$$
\mathbf{j}(\mathbf{r}, t)=-\Gamma \rho(\mathbf{r}, t) \nabla \mu(\mathbf{r}, t),
$$

where $\Gamma$ is a mobility constant. An expression for $\mu$ is obtained within DFT by assuming that, as in the case of the equilibrium fluid, the chemical potential is given by the functional derivative of the Helmholtz free energy functional with respect to the density profile., ${ }^{5,6}$ This assumption, together with Eq. (1) and the continuity equation

$$
\frac{\partial \rho(\mathbf{r}, t)}{\partial t}=-\nabla \cdot \mathbf{j}(\mathbf{r}, t),
$$

provide a basis for the deterministic formulation of dynamical DFT (DDFT). Equations of this form have been used, for example, to study the dynamics of freezing ${ }^{7}$ and of solvation. ${ }^{8}$ More recently, the more systematic approach of MT has been used with much success to describe the dynamics for several different systems. These applications refer to particles in various external, time-dependent, potentials. ${ }^{9-12}$ For the systems considered, the agreement between theory and the results from Brownian dynamics simulations have generally been very good.

An alternative approach to obtaining a DDFT is to view the fluid one-body density profile [denoted $\bar{\rho}(\mathbf{r}, t)$ in order to distinguish it from $\rho(\mathbf{r}, t)$, the "ensemble" averaged density profile] as some sort of spatial and/or time coarse grained average of the density operator $\hat{\rho}(\mathbf{r}, t)=\sum_{i=1}^{N} \delta\left(\mathbf{r}-\mathbf{r}_{i}(t)\right)$, where the $\mathbf{r}_{i}(t)$ are the positions of the $N$ particles in the system. In this case, for Brownian particles, the equation governing the dynamics of $\bar{\rho}(\mathbf{r}, t)$ will, of course, still contain a stochastic element. This is the viewpoint of a number of theories. ${ }^{13-18}$ There is some confusion in the literature ${ }^{19}$ (see also Ref. 20) as to what precisely is meant by $\rho(\mathbf{r}, t)$. We will attempt to clarify these issues elsewhere. ${ }^{21}$ 
In this paper we adopt the deterministic viewpoint of MT and consider $\rho(\mathbf{r}, t)$ to be an ensemble average, i.e., an average over all realizations of the stochastic noise in the preceding interval, until time $t$. We employ the Smoluchowski equation as the starting point for an alternative derivation of the DDFT for classical fluids developed by MT in Refs. 3 and 4. The present scheme for deriving the DDFT bears some similarity in spirit to that given recently in Ref. 22, where projector-operator techniques are used to obtain first the Smoluchowski equation and subsequently an equation of motion for the fluid one-body density profile-the DDFT. Before proceeding with our derivation of the DDFT, in Sec. II we give a brief introduction to the Smoluchowski equation, expounding some of the physical assumptions concerning the dynamics of the fluid that are implicit in this equation. In Sec. III we proceed with the derivation of the DDFT of MT from the Smoluchowski equation. Section IV describes an important application of the DDFT to analyze the short- and intermediate-time dynamics of spinodal decomposition relevant to colloidal fluids. Finally, in Sec. V we make some concluding remarks.

\section{THE SMOLUCHOWSKI EQUATION}

The Smoluchowski equation ${ }^{23-27}$ is a Fokker-Planck equation (or generalized diffusion equation) for interacting Brownian particles. A physically intuitive way of arriving at this equation, e.g., Ref. 24, proceeds as follows: For a fluid of $N$ Brownian particles, one imagines applying a hypothetical force on the particles, where the force on the $j$ th particle is $\mathbf{F}_{j}=-\nabla_{j} \Psi\left(\mathbf{r}^{N}\right) \quad\left(\mathbf{r}^{N} \equiv\left\{\mathbf{r}_{1}, \mathbf{r}_{2} \cdots \mathbf{r}_{N}\right\}\right.$ is the set of position coordinates for the $N$ particles). The equilibrium probability density function in this situation will be

$$
P\left(\mathbf{r}^{N}\right)=\frac{1}{Z} \exp \left[-\beta \Psi\left(\mathbf{r}^{N}\right)-\beta U\left(\mathbf{r}^{N}\right)\right],
$$

where $Z$ is a normalization factor, $\beta=1 / k_{B} T$ is the inverse temperature, and $U\left(\mathbf{r}^{N}\right)$ is the potential energy due to the interparticle interactions and any other external potentials. Taking the gradient of Eq. (3) we obtain

$$
\mathbf{F}_{j}=\nabla_{j} U\left(\mathbf{r}^{N}\right)+k_{B} T \frac{\nabla_{j} P\left(\mathbf{r}^{N}\right)}{P\left(\mathbf{r}^{N}\right)} .
$$

If $\mathbf{F}_{j}$ is switched off, there will be a force $-\mathbf{F}_{j}$ driving the diffusion of particle $j$. We now assume that for time scales $\gg \tau_{B}$, the Brownian time scale, the velocity of the $i$ th particle is

$$
\mathbf{v}_{i}=-\sum_{j=1}^{N} \boldsymbol{\Gamma}_{i j} \cdot \mathbf{F}_{j}
$$

where $\boldsymbol{\Gamma}_{i j}=\beta \mathbf{D}_{i j}\left(\mathbf{r}^{N}\right)$ is the mobility tensor and $\mathbf{D}_{i j}$ is the diffusion tensor. We also assume that we can use the expression in Eq. (4) for the force $\mathbf{F}_{j}$ in (5), with the equilibrium probability density function $P\left(\mathbf{r}^{N}\right)$ replaced by the nonequilibrium probability density function $P\left(\mathbf{r}^{N}, t\right)$ and $U\left(\mathbf{r}^{N}\right)$ replaced by $U\left(\mathbf{r}^{N}, t\right)$ (i.e., there may be a one-body timedependent external potential). It is implicitly assumed that as the particles interact, the momentum degrees of freedom equilibrate much faster than the positional degrees of free- dom, and we have effectively averaged over the momentum degrees of freedom, while keeping the particle coordinates fixed. For a colloidal fluid, this thermal equilibration should occur via the solvent, and this approximation should be a good one to make. For atomic fluids, this may not be the case, especially for particles interacting via harshly repulsive (hard-sphere-like) potentials. For fluids interacting with softer potentials, such as in the Gaussian core model, ${ }^{9}$ this might be a reasonable approximation to make, particularly when the fluid is at high densities where each particle interacts with a large number of neighbors and so the momentum degrees of freedom can equilibrate faster. Since the particle number is conserved, we can expect the fluid to obey the continuity equation

$$
\frac{\partial P\left(\mathbf{r}^{N}, t\right)}{\partial t}=-\sum_{i=1}^{N} \nabla_{i} \cdot\left[\mathbf{v}_{i} P\left(\mathbf{r}^{N}, t\right)\right] .
$$

Substituting Eqs. (4) and (5) into (6), one finds

$\frac{\partial P\left(\mathbf{r}^{N}, t\right)}{\partial t}=\sum_{i=1}^{N} \sum_{j=1}^{N} \nabla_{i} \cdot \boldsymbol{\Gamma}_{i j} \cdot\left[k_{B} T \nabla_{j}+\nabla_{j} U\left(\mathbf{r}^{N}, t\right)\right] P\left(\mathbf{r}^{N}, t\right)$.

If the potential energy term $U\left(\mathbf{r}^{N}, t\right)=0$, then $\boldsymbol{\Gamma}_{i j}=\Gamma \delta_{i j}$ $=\beta D \delta_{i j}$, where $D$ is the diffusion coefficient and Eq. (7) reduces to the diffusion equation, $(\partial / \partial t) P\left(\mathbf{r}^{N}, t\right)$ $=\beta \Gamma \Sigma_{i} \nabla_{i}^{2} P\left(\mathbf{r}^{N}, t\right)$. For a system of interacting particles the diffusion tensor does not, in general, take such a simple form. ${ }^{24,28}$ However, if we neglect the hydrodynamic interactions, we can replace $\boldsymbol{\Gamma}_{i j}$ by its mean-field value, $\Gamma \delta_{i j}$, and then Eq. (7) reduces to a generalized diffusion equation, termed the Smoluchowski equation,

$$
\frac{\partial P\left(\mathbf{r}^{N}, t\right)}{\partial t}=\Gamma \sum_{i=1}^{N} \nabla_{i}\left[k_{B} T \nabla_{i}+\nabla_{i} U\left(\mathbf{r}^{N}, t\right)\right] P\left(\mathbf{r}^{N}, t\right) .
$$

More formally, the Smoluchowski equation is the FokkerPlanck equation for a system of $N$ Brownian particles in the large friction limit. ${ }^{23,26,27}$ The Langevin equation for a system of $N$ Brownian particles of mass $m$ is

$$
m \frac{\mathrm{d}^{2} \mathbf{r}_{i}(t)}{\mathrm{d} t^{2}}+\Gamma^{-1} \frac{\mathrm{d} \mathbf{r}_{i}(t)}{\mathrm{d} t}=-\nabla_{i} U\left(\mathbf{r}^{N}, t\right)+\mathbf{X}_{i}(t),
$$

where $\mathbf{X}_{i}(t)=\left(\xi_{i}^{x}(t), \xi_{i}^{y}(t), \xi_{i}^{z}(t)\right)$ is a white noise term with the property $\left\langle\xi_{i}^{\alpha}(t)\right\rangle=0$ and $\left\langle\xi_{i}^{\alpha}(t) \xi_{i}^{\nu}\left(t^{\prime}\right)\right\rangle=2 k_{B} T \delta_{i j} \delta^{\alpha \nu} \delta(t$ $\left.-t^{\prime}\right)$. When the friction constant $\Gamma^{-1}$ is large, we may neglect the second derivative with respect to time in Eq. (9), and we obtain the stochastic equation of motion, ${ }^{3,4}$

$$
\frac{\mathrm{d} \mathbf{r}_{i}(t)}{\mathrm{d} t}=-\Gamma \nabla_{i} U\left(\mathbf{r}^{N}, t\right)+\Gamma \mathbf{X}_{i}(t) .
$$

The (generalized) Fokker-Planck equation for the distribution function $P\left(\mathbf{r}^{N}, t\right)$ corresponding to this Langevin equation is Eq. (8). ${ }^{23,26,27}$

\section{DYNAMICS OF THE ONE-BODY DENSITY PROFILE}

In this section we derive an equation for the time evolution of the one-body density profile, $\rho(\mathbf{r}, t)$, from the Smoluchowski equation, Eq. (8). For a similar approach based 
solely on pair potentials see Refs. 24,29 , and 30 . The onebody density is merely the integral of the probability distribution function,

$$
\rho\left(\mathbf{r}_{1}, t\right)=N \int \mathrm{d} \mathbf{r}_{2} \cdots \int \mathrm{d} \mathbf{r}_{N} P\left(\mathbf{r}^{N}, t\right) .
$$

Similarly, the two-body density is

$$
\rho^{(2)}\left(\mathbf{r}_{1}, \mathbf{r}_{2}, t\right)=N(N-1) \int \mathrm{d} \mathbf{r}_{3} \cdots \int \mathrm{d} \mathbf{r}_{N} P\left(\mathbf{r}^{N}, t\right),
$$

and in general the $n$-particle density is

$$
\rho^{(n)}\left(\mathbf{r}^{n}, t\right)=\frac{N !}{(N-n) !} \int \mathrm{d} \mathbf{r}_{n+1} \cdots \int \mathrm{d} \mathbf{r}_{N} P\left(\mathbf{r}^{N}, t\right) .
$$

Using Eqs. (11)-(13), and assuming that the potential energy function can be expressed in terms of a one-body external potential acting on each particle, $V_{\text {ext }}\left(\mathbf{r}_{i}, t\right)$, and that the particle interactions are a sum of pair potentials, $v_{2}\left(\mathbf{r}_{i}, \mathbf{r}_{j}\right)$, three-body potentials $v_{3}\left(\mathbf{r}_{i}, \mathbf{r}_{j}, \mathbf{r}_{k}\right)$, and higher body interactions,

$$
\begin{aligned}
U\left(\mathbf{r}^{N}, t\right)= & \sum_{i=1}^{N} V_{\mathrm{ext}}\left(\mathbf{r}_{i}, t\right)+\frac{1}{2} \sum_{j \neq i} \sum_{i=1}^{N} v_{2}\left(\mathbf{r}_{i}, \mathbf{r}_{j}\right) \\
& +\frac{1}{6} \sum_{k \neq j \neq i} \sum_{j \neq i} \sum_{i=1}^{N} v_{3}\left(\mathbf{r}_{i}, \mathbf{r}_{j}, \mathbf{r}_{k}\right)+\cdots
\end{aligned}
$$

then we find that on integrating Eq. (8), one obtains

$$
\begin{aligned}
\Gamma^{-1} \frac{\partial \rho\left(\mathbf{r}_{1}, t\right)}{\partial t}= & k_{B} T \nabla_{1}^{2} \rho\left(\mathbf{r}_{1}, t\right)+\nabla_{1}\left[\rho\left(\mathbf{r}_{1}, t\right) \nabla_{1} V_{\text {ext }}\left(\mathbf{r}_{1}, t\right)\right] \\
& +\nabla_{1} \int \mathrm{d} \mathbf{r}_{2} \rho^{(2)}\left(\mathbf{r}_{1}, \mathbf{r}_{2}, t\right) \nabla_{1} v_{2}\left(\mathbf{r}_{1}, \mathbf{r}_{2}\right) \\
& +\nabla_{1} \cdot \int \mathrm{d} \mathbf{r}_{2} \int \mathrm{d} \mathbf{r}_{3} \rho^{(3)}\left(\mathbf{r}_{1}, \mathbf{r}_{2}, \mathbf{r}_{3}, t\right) \\
& \times \nabla_{1} v_{3}\left(\mathbf{r}_{1}, \mathbf{r}_{2}, \mathbf{r}_{3}\right)+\cdots .
\end{aligned}
$$

We note that if $\partial \rho(\mathbf{r}, t) / \partial t=0$, then Eq. (15) is equivalent to the derivative of the first equation of the YBG hierarchy. ${ }^{31}$

For a fluid in equilibrium, there is an exact sum rule ${ }^{5}$ which relates the gradient of the one-body direct correlation function to the interparticle forces acting on a particle [recall that $-k_{B} T c^{(1)}(\mathbf{r})$ is the effective one-body potential due to interactions in the fluid]. If the particles interact solely via pair potentials: $-k_{B} T \rho(\mathbf{r}) \nabla c^{(1)}(\mathbf{r})=\int \mathrm{d} \mathbf{r}^{\prime} \rho^{(2)}\left(\mathbf{r}, \mathbf{r}^{\prime}\right)$ $\times \nabla v_{2}\left(\mathbf{r}, \mathbf{r}^{\prime}\right)$. This result can be generalized straightforwardly to fluids where the particles interact via many-body potentials, Eq. (14), giving

$$
\begin{aligned}
-k_{B} T & \rho\left(\mathbf{r}_{1}\right) \nabla c^{(1)}\left(\mathbf{r}_{1}\right) \\
= & \int \mathrm{d} \mathbf{r}_{2} \rho^{(2)}\left(\mathbf{r}_{1}, \mathbf{r}_{2}\right) \nabla v_{2}\left(\mathbf{r}_{1}, \mathbf{r}_{2}\right) \\
& +\int \mathrm{d} \mathbf{r}_{2} \int \mathrm{d} \mathbf{r}_{3} \rho^{(3)}\left(\mathbf{r}_{1}, \mathbf{r}_{2}, \mathbf{r}_{3}\right) \nabla_{1} v_{3}\left(\mathbf{r}_{1}, \mathbf{r}_{2}, \mathbf{r}_{3}\right)+\cdots \\
= & \sum_{n=2}^{\infty} \int \mathrm{d} \mathbf{r}_{2} \cdots \int \mathrm{d} \mathbf{r}_{n} \rho^{(n)}\left(\mathbf{r}^{n}\right) \nabla_{1} v_{n}\left(\mathbf{r}^{n}\right) .
\end{aligned}
$$

From equilibrium statistical mechanics one also knows that $c^{(1)}(\mathbf{r})$ is equal to the functional derivative of the excess (over ideal) part of the Helmholtz free energy functional, ${ }^{5}$

$$
c^{(1)}(\mathbf{r})=-\beta \frac{\delta F_{\mathrm{ex}}[\rho(\mathbf{r})]}{\delta \rho(\mathbf{r})},
$$

evaluated at the equilibrium density. [Generally $c^{(1)}(\mathbf{r})$ is a functional of $\rho(\mathbf{r})$.] Making the approximation that these identities, Eqs. (16) and (17), valid for the equilibrium fluid, hold also for the nonequilibrium fluid and substituting into Eq. (15), we obtain the DDFT equation, stated without justification by Evans [see Eqs. (166) and (167) of Ref. 5], and derived much more convincingly, for the case of fluids interacting solely via pair potentials, by $\mathrm{MT}^{3,4}$ i.e.,

$$
\Gamma^{-1} \frac{\partial \rho(\mathbf{r}, t)}{\partial t}=\nabla\left[\rho(\mathbf{r}, t) \nabla \frac{\delta F[\rho(\mathbf{r}, t)]}{\delta \rho(\mathbf{r}, t)}\right],
$$

where $F[\rho(\mathbf{r}, t)]$ is the Helmholtz free energy functional,

$$
\begin{aligned}
F[\rho(\mathbf{r}, t)]= & k_{B} T \int \mathrm{d} \mathbf{r} \rho(\mathbf{r}, t)\left[\ln \left(\rho(\mathbf{r}, t) \Lambda^{3}\right)-1\right] \\
& +F_{\mathrm{ex}}[\rho(\mathbf{r}, t)]+\int \mathrm{d} \mathbf{r} V_{\mathrm{ext}}(\mathbf{r}, t) \rho(\mathbf{r}, t) .
\end{aligned}
$$

The first term is the ideal gas free energy; $\Lambda$ is the de Broglie wavelength. In obtaining Eq. (18) by using Eqs. (16) and (17), which are strictly equilibrium results, we are effectively assuming that the correlations between the particles when the fluid is out of equilibrium are equivalent to those for an equilibrium fluid with the same one-body density profile $\rho(\mathbf{r}, t)$.

We can obtain further insight into the status of Eq. (18) by rewriting it as follows:

$$
\Gamma^{-1} \frac{\partial \rho(\mathbf{r}, t)}{\partial t}=\nabla[\rho(\mathbf{r}, t) \nabla \mu(\mathbf{r}, t)],
$$

where $-\nabla \mu(\mathbf{r}, t) \equiv-\nabla(\delta F[\rho] / \delta \rho)$ is the net driving force acting on a particle located at $(\mathbf{r}, t)$. The chemical potential obtained from Eq. (19) has three contributions,

$$
\frac{\delta F[\rho(\mathbf{r}, t)]}{\delta \rho(\mathbf{r}, t)} \equiv \mu(\mathbf{r}, t)=\mu_{\mathrm{id}}(\mathbf{r}, t)+\mu_{\mathrm{int}}(\mathbf{r}, t)+\mu_{\mathrm{ext}}(\mathbf{r}, t) .
$$

The first contribution is the ideal gas entropic term $\mu_{\mathrm{id}}(\mathbf{r}, t)$ $=k_{B} T \ln \Lambda^{3} \rho(\mathbf{r}, t)$, the second contribution, $\mu_{\text {int }}(\mathbf{r}, t)$ $=-k_{B} T c^{(1)}(\mathbf{r})$, is that due to the interactions with the other particles in the fluid, and the final term is simply the external potential, $\mu_{\text {ext }}(\mathbf{r}, t)=V_{\text {ext }}(\mathbf{r}, t)$. As noted by MT, ${ }^{3}$ for noninteracting particles, $\mu_{\text {int }} \equiv 0$, and Eq. (20) reduces to the exact equation for the diffusion of an ideal Brownian gas, i.e.,

$$
\Gamma^{-1} \frac{\partial \rho(\mathbf{r}, t)}{\partial t}=k_{B} T \nabla^{2} \rho(\mathbf{r}, t)+\nabla\left[\rho(\mathbf{r}, t) \nabla V_{\mathrm{ext}}(\mathbf{r}, t)\right] .
$$

For an inhomogeneous interacting fluid in equilibrium the density profile satisfies ${ }^{5}$

$$
\frac{\delta F[\rho(\mathbf{r})]}{\delta \rho(\mathbf{r})}=\mu=\mathrm{constant}
$$


where the Helmholtz free energy functional $F[\rho]$ is given by Eq. (19) with $\rho(\mathbf{r}, t)$ replaced by $\rho(\mathbf{r})$ and $V_{\text {ext }}(\mathbf{r}, t)$ replaced by $V_{\text {ext }}(\mathbf{r})$, so there is no net driving force on the particles. It follows that for a time-independent external potential, $V_{\text {ext }}(\mathbf{r}, t) \rightarrow V_{\text {ext }}(\mathbf{r})$, as $t \rightarrow \infty$, regardless of the initial profile $\rho(\mathbf{r}, t=0)$, Eq. (18) will yield the same one-body density profile in the limit $t \rightarrow \infty$ as does the equilibrium DFT [i.e., the solution to Eq. (23)] for the same external potential. The present derivation provides, we believe, additional insight to that of $\mathrm{MT}^{3,4}$ into the physics incorporated into Eq. (18) (the key DDFT equation). Assuming one has an accurate expression for the Helmholtz free energy functional Eq. (19), and in particular for the excess Helmholtz free energy functional, Eq. (18) should provide an accurate description of the dynamics of $\rho(\mathbf{r}, t)$ for a system of Brownian particles.

\section{SPINODAL DECOMPOSITION FROM THE DDFT EQUATION}

In this section we apply the DDFT derived in the preceding section to fluid spinodal decomposition. Since the basis for the DDFT is the Smoluchowski equation, an equation of motion for Brownian particles, we expect our theory to be particularly relevant to spinodal decomposition in colloidal fluids, rather than molecular fluids. In colloidal fluids friction with the solvent results in a much faster equilibration of the momentum degrees of freedom compared with those of the particle positions. However, since we do not explicitly include the particles of the solvent in which the colloids are suspended, our theory neglects the hydrodynamic interactions that may be significant to spinodal decomposition in some colloidal fluids.

When a (colloidal) fluid which exhibits liquid-gas phase separation (or more generally fluid-fluid phase separation) is quenched to a state point in the region of the phase diagram where there is coexistence, the fluid can phase separate in two distinct ways. The first mechanism is that which occurs when the state point to which the fluid is quenched is near to the binodal. In this case phase separation generally occurs via nucleation of droplets of one phase forming in the other phase. ${ }^{32,33}$ For example, if the fluid is quenched to a state point inside the binodal on the liquid side, then bubbles of the gas phase can nucleate in the bulk of the metastable liquid.

However, if the fluid is quenched to a state point well inside the binodal, then a different mechanism for phase separation is possible: spinodal decomposition. Spinodal decomposition is characterized by the exponential growth of density fluctuations of certain wavelengths. ${ }^{32,33}$ In (meanfield) theoretical descriptions, liquid-gas phase separation is determined by the occurrence of a van der Waals loop in the Helmholtz free energy per particle, $f(v)$, where $v$ is the volume per particle. Spinodal decomposition is predicted to occur in regions of the phase diagram where $\left(\partial^{2} f / \partial v^{2}\right)_{T}$ $<0$, i.e., regions where the isothermal compressibility $\chi_{T}$ is predicted to be negative. The boundary to this region, the spinodal, is defined by the locus of $\left(\partial^{2} f / \partial v^{2}\right)_{T}=0$ in the phase diagram. Experimentally there is not necessarily a sharp distinction between regions where phase separation occurs via nucleation and via spinodal decomposition. How-

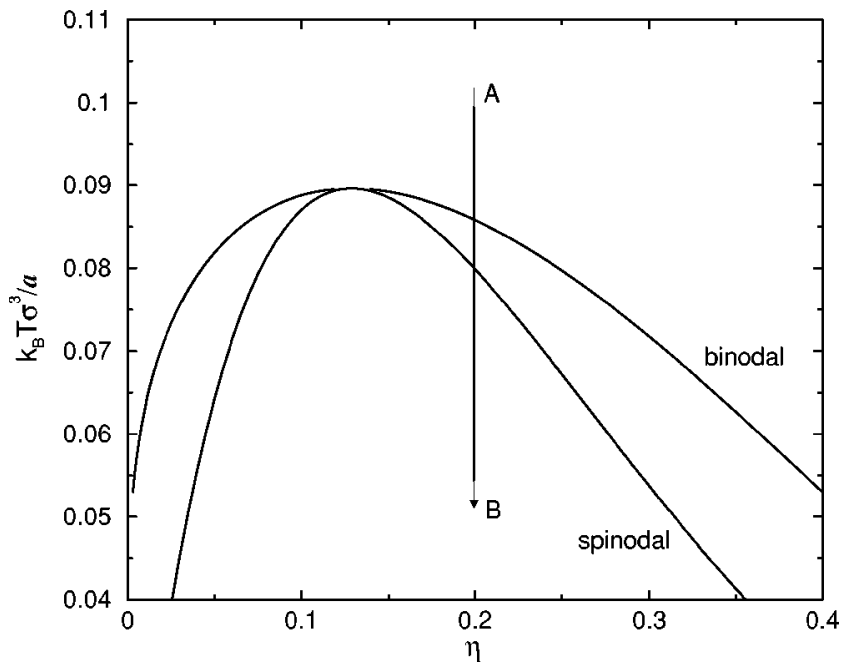

FIG. 1. Phase diagram for a fluid composed of particles interacting via pair potentials of the form in Eqs. (38)-(40), calculated from the free energy of Eq. (41). $\eta=\pi \rho_{b} \sigma^{3} / 6$ is the packing fraction and $k_{B} T \sigma^{3} / a$ is the (reduced) temperature. The path from $A$ to $B$ denotes the quench corresponding to the results in Figs. 2 and 3.

ever, in a deep quench far from the spinodal, spinodal decomposition is the mechanism generally expected for phase separation.

In a fluid undergoing spinodal decomposition three different regimes can be distinguished. For early times after the quench, the amplitude of the density fluctuations are small and theories linear in the density fluctuations such as the well-known Cahn-Hilliard theory ${ }^{34,35}$ (see also Refs. 29, 32, 33,36 , and 37) provide a good description of this (early) stage of spinodal decomposition. At intermediate times the density fluctuations can be large, but sharp interfaces between domains of gaslike and liquidlike regions have still not formed. ${ }^{29}$ At later stages there are sharp interfaces between domains of liquid and gas and successful theoretical descriptions of the later stage dynamics of spinodal decomposition, such as the Allen-Cahn theory ${ }^{38}$ (see also Refs. 32, 33, and 39), focus on the dynamics of the interfaces.

First, in Sec. IV A, we shall use the DDFT formalism to derive a generalization of the (linear) Cahn-Hilliard theory, ${ }^{34,35}$ similar to that described in Refs. 29, 36, and 37, for the early stages of spinodal decomposition, when the density fluctuations are small. Then in Sec. IV B we will proceed to derive a nonlinear theory which we believe may be applicable for the dynamics of spinodal decomposition at both short and intermediate time scales. Results of explicit calculations for a model fluid are given in Sec. IV C.

\section{A. Early stages of spinodal decomposition}

We consider spinodal decomposition in the bulk of a fluid, so we set $V_{\text {ext }}(\mathbf{r}, t)=0$ in Eqs. (18) and (19) and consider small density fluctuations $\widetilde{\rho}(\mathbf{r}, t)=\rho(\mathbf{r}, t)-\rho_{b}$ about the bulk fluid density, $\rho_{b}$, i.e., we are considering a homogeneous fluid which has been rapidly quenched to the region of the phase diagram inside the spinodal (e.g., from $A$ to $B$ in Fig. 1) and we seek those wave numbers $k$ for which density fluctuations grow. From Eqs. (17), (18), and (19) we obtain 


$$
\begin{aligned}
\left(\Gamma k_{B} T\right)^{-1} \frac{\partial \widetilde{\rho}(\mathbf{r}, t)}{\partial t}= & \nabla^{2} \widetilde{\rho}(\mathbf{r}, t)-\rho_{b} \nabla^{2} c^{(1)}(\mathbf{r}, t) \\
& -\nabla\left[\widetilde{\rho}(\mathbf{r}, t) \nabla c^{(1)}(\mathbf{r}, t)\right] .
\end{aligned}
$$

This approach is basically that of Refs. 5 and 36. In Ref. 5 Evans writes down Eq. (18), and then linearizes Eq. (24) in $\widetilde{\rho}$ by Taylor expanding $c^{(1)}$ about the bulk fluid value, giving

$$
c^{(1)}(\mathbf{r})=c^{(1)}(\infty)+\left.\int \mathrm{d} \mathbf{r}^{\prime} \frac{\delta c^{(1)}(\mathbf{r})}{\delta \rho\left(\mathbf{r}^{\prime}\right)}\right|_{\rho_{b}} \widetilde{\rho}\left(\mathbf{r}^{\prime}, t\right)+\mathcal{O}\left(\widetilde{\rho}^{2}\right),
$$

where $c^{(1)}(\infty) \equiv c^{(1)}\left[\rho_{b}\right]=-\beta \mu_{\mathrm{ex}}$ and $\mu_{\mathrm{ex}}$ is the excess chemical potential. The second term simplifies by recalling ${ }^{5}$

$$
\begin{aligned}
\frac{\delta c^{(1)}(\mathbf{r})}{\delta \rho\left(\mathbf{r}^{\prime}\right)} & =-\beta \frac{\delta^{2} F_{\mathrm{ex}}[\rho]}{\delta \rho\left(\mathbf{r}^{\prime}\right) \delta \rho(\mathbf{r})} \\
& =c^{(2)}\left(\mathbf{r}, \mathbf{r}^{\prime}\right)=c^{(2)}\left(\left|\mathbf{r}-\mathbf{r}^{\prime}\right| ; \rho_{b}\right),
\end{aligned}
$$

for a homogeneous fluid of spherically symmetric particles. For an equilibrium system $c^{(2)}\left(r ; \rho_{b}\right)$ is the OrnsteinZernike pair direct correlation function of the fluid of density $\rho_{b}$. Substituting Eq. (25) into Eq. (24), keeping only terms that are linear in the fluctuation $\widetilde{\rho}$, we obtain. ${ }^{5,36}$

$$
\begin{aligned}
& \left(\Gamma k_{B} T\right)^{-1} \frac{\partial \widetilde{\rho}(\mathbf{r}, t)}{\partial t} \\
& \quad=\nabla^{2} \widetilde{\rho}(\mathbf{r}, t)-\rho_{b} \nabla^{2}\left[\int \mathrm{d} \mathbf{r}^{\prime} c^{(2)}\left(\left|\mathbf{r}-\mathbf{r}^{\prime}\right| ; \rho_{b}\right) \widetilde{\rho}\left(\mathbf{r}^{\prime}, t\right)\right] .
\end{aligned}
$$

Fourier transforming yields an equation for the time evolution of the different Fourier components

$$
\hat{\rho}(\mathbf{k}, t)=\int \mathrm{d} \mathbf{r} \exp (i \mathbf{k} \cdot \mathbf{r}) \widetilde{\rho}(\mathbf{r}, t),
$$

and one obtains

$$
\left(\Gamma k_{B} T\right)^{-1} \frac{\partial \hat{\rho}(k, t)}{\partial t}=-k^{2} \hat{\rho}(k, t)+\rho_{b} k^{2} \hat{c}(k) \hat{\rho}(k, t),
$$

where $\hat{c}(k)=\int \mathrm{d} \mathbf{r} \exp (i \mathbf{k} \cdot \mathbf{r}) c^{(2)}\left(r ; \rho_{b}\right)$. The solution of Eq. (29) is

$$
\hat{\rho}(k, t)=\hat{\rho}(k, 0) \exp [R(k) t],
$$

where $R(k)=-\Gamma k_{B} T k^{2}\left(1-\rho_{b} \hat{c}(k)\right)$. For an equilibrium fluid, at a state point outside the spinodal, $S(k) \equiv(1$ $\left.-\rho_{b} \hat{c}(k)\right)^{-1}$ is the usual static structure factor ${ }^{31,40}$ and, since for an equilibrium fluid $S(k)>0$ for all values of $k$, it follows that outside the spinodal $R(k)<0$ for all values of $k$. From Eq. (30), all Fourier components will decay implying, of course, that the fluid is stable.

On approaching the spinodal from the single phase region one finds that $S(k=0) \rightarrow \infty$; at the spinodal (1 $\left.-\rho_{b} \hat{c}(k=0)\right)=0$. Within the mean-field (van der Waalstype) theory of fluids to be described below, we find that inside the spinodal $S(k)$ can take negative values ${ }^{40}$ and thus the quantity $R(k)$ can have positive values for $k<k_{c}$, where the value of $k_{c}$ depends upon how far into the spinodal region one has quenched, see for example, Fig. 2, which em-

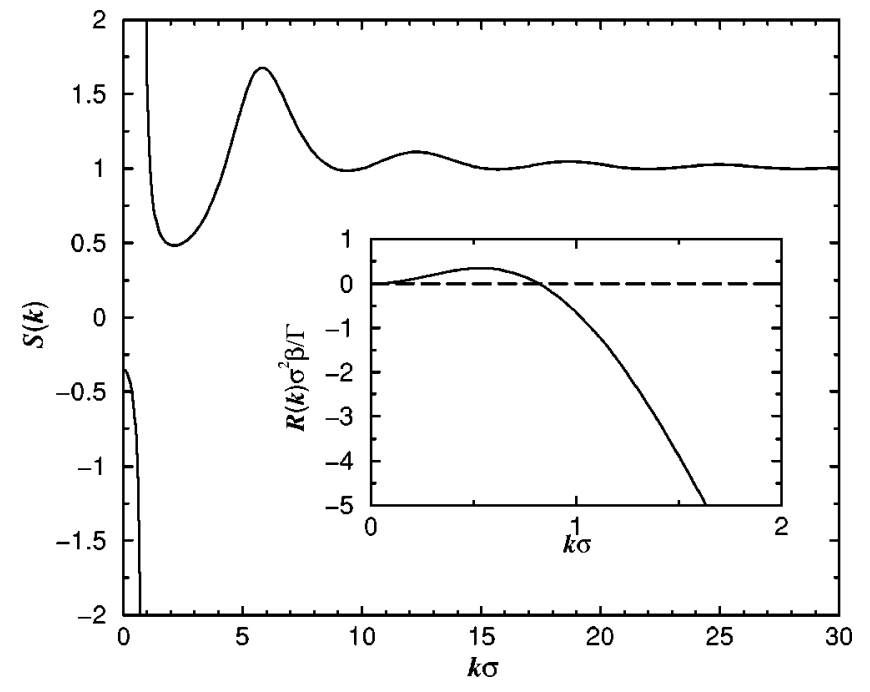

FIG. 2. The function $S(k) \equiv\left(1-\rho_{b} \hat{c}(k)\right)^{-1}$ calculated using Eq. (43) at the state point (inside the spinodal) with $\eta=0.2$ and $k_{B} T \sigma^{3} / a=0.05$, point $B$ in Fig. 1. Note that for $k<k_{c}$, where $k_{c} \sigma \simeq 0.8, S(k)<0$. In the inset we plot $R(k) \sigma^{2} \beta / \Gamma=-k^{2} \sigma^{2} / S(k)$, the factor appearing in the exponential in Eq. (30). In the initial stages of spinodal decomposition density fluctuations with wave numbers $k<k_{c}$ grow exponentially, whereas for $k>k_{c}$ the fluctuations are damped.

ploys a particular approximation, namely Eq. (43), for $\hat{c}(k)$. $k_{c}$ is obtained as the solution to the equation $\rho_{b} \hat{c}\left(k_{c}\right)=1$. Thus we find that inside the spinodal region there will be some density fluctuations with a wave number $k<k_{c}$ whose amplitude will grow exponentially. ${ }^{5,29,30,34-37}$ The deeper the quench into the spinodal region, the larger the value of $k_{c}$ can be.

This general picture of the exponential growth of certain Fourier components (modes) was obtained originally by Cahn and Hilliard ${ }^{34,35}$ who derived an explicit approximation for the function $R(k)$. Cahn-Hilliard theory for spinodal decomposition is usually derived by considering the continuity equation (2), together with the following approximation for the current:

$$
\mathbf{j}(\mathbf{r}, t)=-M \nabla \frac{\delta F[\rho(\mathbf{r}, t)]}{\delta \rho(\mathbf{r}, t)},
$$

where $M$ is a mobility constant and the functional $F$ is chosen to be of the $\phi^{4}$ Ginzburg-Landau form. Generalizing slightly we assume that the free energy functional has the square-gradient form ${ }^{5}$

$$
F_{\mathrm{sg}}[\rho(\mathbf{r}, t)]=\int \mathrm{d} \mathbf{r}\left[f_{0}(\rho(\mathbf{r}, t))+\frac{1}{2} K|\nabla \rho(\mathbf{r}, t)|^{2}\right],
$$

where $f_{0}(\rho) \equiv \rho f(\rho)$ is the Helmholtz free energy density for the homogeneous fluid of density $\rho$ and we treat $K$ as a positive constant. From Eq. (2) one then finds

$$
\frac{\partial \rho(\mathbf{r}, t)}{\partial t}=M \nabla^{2}\left[\frac{\partial f_{0}(\rho(\mathbf{r}, t))}{\partial \rho(\mathbf{r}, t)}-K \nabla^{2} \rho(\mathbf{r}, t)\right] .
$$

This equation is then linearized about the bulk density $\rho_{b}$ to obtain $^{32,36}$ 


$$
\frac{\partial \widetilde{\rho}(\mathbf{r}, t)}{\partial t}=M \nabla^{2}\left[\left(\frac{\partial^{2} f_{0}}{\partial \rho^{2}}\right)_{\rho_{b}}-K \nabla^{2}\right] \widetilde{\rho}(\mathbf{r}, t) .
$$

On Fourier transforming Eq. (34) one obtains $\partial \hat{\rho}(k, t) / \partial t$ $=R_{\mathrm{sg}}(k) \hat{\rho}(k, t), \quad$ where $\quad R_{\mathrm{sg}}(k)=-M k^{2}\left(K k^{2}+\left(\partial^{2} f_{0} /\right.\right.$ $\partial \rho^{2} \rho_{\rho_{b}}$ ), the solution to which is Eq. (30), with $R(k)$ replaced by $R_{\mathrm{sg}}(k)$. Note that $\left(\partial^{2} f_{0} / \partial \rho^{2}\right)_{\rho_{b}}$ is negative inside the spinodal. It is clear from this argument that CahnHilliard theory can therefore be viewed as a special case of the more general linear theory presented in the earlier part of this section. ${ }^{41}$ By employing a free energy functional more accurate than (32) one should be able to incorporate short wavelength density fluctuations as well as the long wavelength, $k \rightarrow 0$, fluctuations accounted for by a square-gradient approach.

\section{B. Spinodal decomposition at intermediate times}

In order to go one step beyond the lowest order (linear) theories described above, we consider an approximate excess Helmholtz free energy functional, obtained by Taylor expanding the excess Helmholtz free energy about the uniform density. By integrating Eq. (25) and omitting terms beyond $\mathcal{O}\left(\tilde{\rho}^{2}\right)$ we obtain

$$
\begin{aligned}
F_{\mathrm{ex}}[\rho]= & F_{\mathrm{ex}}\left[\rho_{b}\right]+\mu_{\mathrm{ex}} \int \mathrm{d} \mathbf{r} \widetilde{\rho}(\mathbf{r}, t) \\
& -\frac{k_{B} T}{2} \int \mathrm{d} \mathbf{r} \int \mathrm{d} \mathbf{r}^{\prime} \widetilde{\rho}(\mathbf{r}, t) \widetilde{\rho}\left(\mathbf{r}^{\prime}, t\right) c^{(2)}\left(\left|\mathbf{r}-\mathbf{r}^{\prime}\right| ; \rho_{b}\right) .
\end{aligned}
$$

This truncated quadratic density expansion is often used in the theory of inhomogeneous fluids in equilibrium. ${ }^{1}$ Using Eq. (35) in Eq. (24) we find

$$
\begin{aligned}
\left(\Gamma k_{B} T\right)^{-1} \frac{\partial \widetilde{\rho}(\mathbf{r}, t)}{\partial t}= & \nabla^{2} \widetilde{\rho}(\mathbf{r}, t)-\nabla\left[\left(\rho_{b}+\widetilde{\rho}(\mathbf{r}, t)\right)\right. \\
& \left.\times \int \mathrm{d} \mathbf{r}^{\prime} \widetilde{\rho}\left(\mathbf{r}^{\prime}, t\right) \nabla c^{(2)}\left(\left|\mathbf{r}-\mathbf{r}^{\prime}\right| ; \rho_{b}\right)\right] .
\end{aligned}
$$

The first two terms on the right-hand side are those entering the linear theory (27) while the third term is the only nonlinear one which arises for the functional (35). Fourier transforming Eq. (36) we obtain

$$
\begin{aligned}
\left(\Gamma k_{B} T\right)^{-1} & \frac{\partial \hat{\rho}(k, t)}{\partial t} \\
= & -k^{2} \hat{\rho}(k, t)+\rho_{b} k^{2} \hat{c}(k) \hat{\rho}(k, t) \\
& +\frac{1}{(2 \pi)^{3}} \int \mathrm{d} \mathbf{k}^{\prime} \mathbf{k} \cdot \mathbf{k}^{\prime} \hat{\rho}\left(k^{\prime}, t\right) \hat{c}\left(k^{\prime}\right) \hat{\rho}\left(\left|\mathbf{k}-\mathbf{k}^{\prime}\right|, t\right),
\end{aligned}
$$

which should be compared with Eq. (29); now there is an additional term on the right-hand side is nonlinear in $\hat{\rho}(k, t)$. This term describes the coupling between the different Fourier components of the density fluctuations (modes). ${ }^{42} \mathrm{An}$ equation almost equivalent to Eq. (37) was derived recently by considering the mobility $M$ to be a linear function of the order parameter, rather than a constant, in a Cahn-Hilliard treatment-see Eq. (10) in Ref. 43; we shall discuss this further in Sec. IV D. In order to proceed further we must assume a particular form for the Helmholtz free energy functional, from which we can obtain $\hat{c}(k)$ and thus solve Eq. (37) numerically.

\section{Results for a model fluid}

We consider a fluid composed of particles interacting via pair potentials of the form

$$
v_{2}(r)=v_{\mathrm{hs}}(r)+v_{\mathrm{at}}(r),
$$

where

$$
v_{\text {hs }}(r)=\left\{\begin{array}{cc}
\infty, & r \leqslant \sigma \\
0, & r>\sigma
\end{array}\right.
$$

is the hard-sphere pair potential, and the attractive part of the pair potential has a Yukawa form

$$
v_{\mathrm{at}}(r)=-\frac{a \lambda^{3} \exp (-\lambda r)}{4 \pi \lambda r},
$$

where $a$ and $\lambda$ are positive constants. Provided the decay length $\lambda^{-1}$ is sufficiently large this model fluid exhibits stable, with respect to freezing, liquid-gas phase separation. Pair potentials of this form are often used as crude models for simple fluids but they could be used to model the effective (depletion) potential between the colloids in a colloidpolymer mixture solution ${ }^{44}$ by choosing the pair potential parameters in Eqs. (39) and (40) to mimic the well-known Asakura-Oosawa potential. ${ }^{44-47}$ In calculations for our model system we approximate the excess Helmholtz free energy functional by

$$
F_{\mathrm{ex}}[\rho]=F_{\mathrm{ex}}^{\mathrm{hs}}[\rho]+\frac{1}{2} \int \mathrm{d} \mathbf{r} \int \mathrm{d} \mathbf{r}^{\prime} \rho(\mathbf{r}, t) \rho\left(\mathbf{r}^{\prime}, t\right) v_{\mathrm{at}}\left(\left|\mathbf{r}-\mathbf{r}^{\prime}\right|\right),
$$

where $F_{\mathrm{ex}}^{\mathrm{hs}}[\rho]$ is the reference hard-sphere Helmholtz excess free energy functional and attractive interactions are treated in a mean-field fashion. ${ }^{1}$ If we employ the Rosenfeld Fundamental Measure Theory ${ }^{48-50}$ for $F_{\text {ex }}^{\mathrm{hs}}[\rho]$, this nonlocal functional generates the Percus-Yevick pair direct correlations functions in a hard-sphere fluid. Thus, using Eq. (26) we obtain the following simple (random phase) approximation

$$
c^{(2)}\left(r ; \rho_{b}\right)=c_{\mathrm{PY}}\left(r ; \rho_{b}\right)-\beta v_{\mathrm{at}}(r),
$$

where $c_{\mathrm{PY}}(r)$ is the Percus-Yevick (PY) approximation ${ }^{31}$ for the hard-sphere pair direct correlation function. With this choice the Fourier transform of $c^{(2)}(r)$ can be carried out analytically

$$
\hat{c}(k)=\hat{c}_{\mathrm{PY}}(k)+\frac{\beta a \lambda^{2}}{\lambda^{2}+k^{2}},
$$

where $\hat{c}_{\mathrm{PY}}(k)$ is given by ${ }^{51}$ 


$$
\begin{aligned}
\hat{c}_{\mathrm{PY}}(k)= & -4 \pi \sigma^{3}\left[\left(\frac{\alpha+2 \beta+4 \gamma}{q^{3}}-\frac{24 \gamma}{q^{5}}\right) \sin (q)\right. \\
& +\left(-\frac{\alpha+\beta+\gamma}{q^{2}}+\frac{2 \beta+12 \gamma}{q^{4}}-\frac{24 \gamma}{q^{6}}\right) \cos (q) \\
& \left.+\left(\frac{24 \gamma}{q^{6}}-\frac{2 \beta}{q^{4}}\right)\right],
\end{aligned}
$$

where $q=k \sigma$, and the coefficients

$$
\begin{aligned}
& \alpha=\frac{(1+2 \eta)^{2}}{(1-\eta)^{4}}, \\
& \beta=\frac{-6 \eta(1+\eta / 2)^{2}}{(1-\eta)^{4}}, \\
& \gamma=\frac{\eta(1+2 \eta)^{2}}{2(1-\eta)^{4}}
\end{aligned}
$$

depend upon $\eta=\pi \rho_{b} \sigma^{3} / 6$, the packing fraction. The phase diagram for our model fluid is displayed in Fig. 1. The liquid-gas binodal is calculated by performing the common tangent construction on the Helmholtz free energy per particle for the bulk fluid, $f\left(\rho_{b}\right)=f_{\mathrm{PY}}\left(\rho_{b}\right)-\rho_{b} a / 2$, obtained from Eq. (41); $f_{\mathrm{PY}}\left(\rho_{b}\right)$ is the PY compressibility approximation for the hard-sphere Helmholtz free energy. ${ }^{31}$ The spinodal is the locus of points for which $\partial^{2} f / \partial v^{2}=0$, where $v$ $=1 / \rho_{b}$. For simplicity we choose to set the inverse length scale parameter in the attractive part of the pair potential equal to the hard-sphere diameter, i.e., $\lambda^{-1}=\sigma$. The quantity $a=\int \mathrm{d} \mathbf{r} v_{\mathrm{at}}(r)$ determines the energy scale. We expect the present model fluid to exhibit a freezing transition for large $\eta$, but we do not consider this here.

We are now in a position to use our approximate form for $\hat{c}(k)$, obtained from Eqs. (43) and (44), together with Eq. (37), to calculate $\hat{\rho}(k, t)$ during the early and intermediate times of spinodal decomposition. We assume that at $t=0$ $\hat{\rho}(k, t)$ takes a small, constant, positive value for all values of $k$ and choose $\hat{\rho}(k, t=0)=10^{-8}$. The later time dynamics are insensitive to the specific choice of value for $\hat{\rho}(k, t=0)$ because for short times, when Eq. (30) describes the dynamics of spinodal decomposition, Fourier components (modes) with wave number $k>k_{c}$ do not grow and are exponentially damped, whereas components with $k<k_{c}$ grow exponentially with the initial value $\hat{\rho}(k, t=0)$ as a prefactor. Thus choosing a different value for $\hat{\rho}(k, t=0)$ is effectively a shift of the time axis. For a quench to the state point $k_{B} T \sigma^{3} / a$ $=0.05$ and $\eta=0.2$ (i.e., the quench to point $B$, in Fig. 1) the early time growth gives a single peak in $\hat{\rho}(k, t)$ at $k \sigma \simeq 0.6$ corresponding to where the maximum of $R(k)$ in Eq. (30) occurs-see the inset to Fig. 2. We find that $R(k)$ obtained using Eqs. (43) and (44) for $\hat{c}(k)$ has a similar form to that extracted from molecular dynamics simulation results for a Lennard-Jones fluid deep inside the spinodal region. ${ }^{52}$ Moreover, the overall shape of $R(k)$ and the values of $k_{c} \sigma$ are in keeping with results for the Lennard-Jones fluid (for similar state points) obtained by Evans and Telo Da Gama, ${ }^{36}$ using a theory equivalent to the present, and by Abraham, ${ }^{37}$ using a perturbation theory approach. We also plot in Fig. 2 the function $S(k) \equiv\left(1-\rho_{b} \hat{c}(k)\right)^{-1}$; the negative portion of this

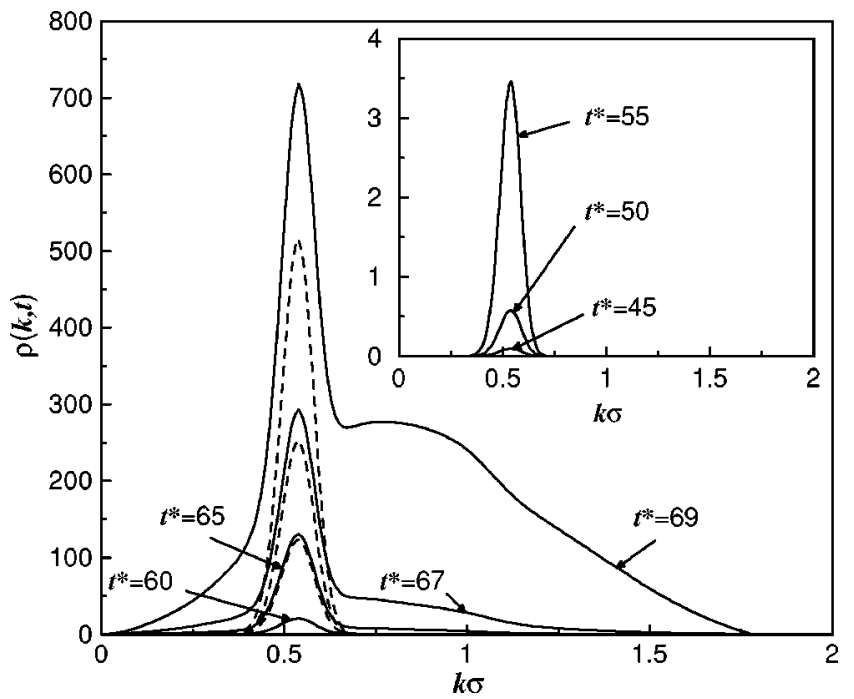

FIG. 3. Plot of $\hat{\rho}(k, t)$ for a quench to the state point $k_{B} T \sigma^{3} / a=0.05$ and $\eta=0.2$, point $B$ in Fig. $1 . \hat{\rho}(k, t)$ is shown for times $t^{*}=k_{B} T \Gamma \sigma^{2} t$ $=45,50,55$ (in the inset) and $t^{*}=60,65,67,69$ (in the main figure). The results obtained from the linear theory [Eq. (29)] are denoted by a dashed line, and those from the nonlinear theory [Eq. (37)] by a solid line. The effect of the coupling between Fourier components (modes) with different wave numbers, described by the nonlinear theory, becomes increasingly significant at later times, whereas for earlier times (see inset), the results from the two theories are indistinguishable.

function corresponds to wave numbers $k$ for which density fluctuations grow exponentially in the early stages of spinodal decomposition.

Some typical plots of $\hat{\rho}(k, t)$ at early times are displayed in the inset to Fig. 3, for a quench to point $B$ in Fig. 1. The plots are for the reduced times $t^{*}=k_{B} T \Gamma \sigma^{2} t=45,50$, and 55 in the inset and 60, 65, 67, and 69 in the main figure. The results obtained from the linear theory, Eq. (29), and the nonlinear theory, Eq. (37), are indistinguishable for the three earliest times (see inset). However, at later times the linear theory (dashed line) continues to give just a single peak in $\hat{\rho}(k, t)$ that grows exponentially, whereas the nonlinear theory (solid line), which includes the effect of coupling between Fourier components with different wave numbers, shows that components with wave numbers different from that predicted by the linear theory can also grow. We see that the effect of the coupling incorporated into the nonlinear theory becomes increasingly significant at intermediate times, producing first a shoulder which grows into a bump in $\hat{\rho}(k, t)$ at a larger wave number, $k \sigma \simeq 0.8$ than that of the main peak which first appears in $\hat{\rho}(k, t)$ at early times.

\section{Discussion}

In simulation studies of spinodal decomposition the quantity that is often measured in order to characterize the fluid is the structure factor

$$
S(k, t)=\frac{1}{N} \sum_{i, j=1}^{N}\left\langle\exp \left(i \mathbf{k}\left[\mathbf{r}_{i}(t)-\mathbf{r}_{j}(t)\right]\right)\right\rangle,
$$

where $\langle\cdots\rangle$ is an ensemble average over different realizations of the stochastic noise in the interval up to time $t$. For small values of $k$ one finds that ${ }^{29}$ 


$$
S(k, t) \simeq A(t)+\frac{1}{N}[\hat{\rho}(k, t)]^{2}
$$

where $A(t)$ is a (small) wave number independent baseline. Thus, to a reasonable approximation $S(k, t) \propto[\hat{\rho}(k, t)]^{2}$ for small values of $k$ (see Refs. 29 and 36). Simulation studies of the early stages of spinodal decomposition in model colloidal fluids such as that in Ref. 53, where the authors consider a fluid composed of particles interacting via the Lennard-Jones pair potential, display the growth of a single peak in $S(k, t)$. This is, of course, a general feature of the very early stages of spinodal decomposition and is found in many other systems. ${ }^{29,32}$ Thus, our results for short times showing the growth of a single peak in $\hat{\rho}(k, t)$, and therefore also in $S(k, t)$, are in keeping with simulation studies.

The development of a second peak (shoulder) in $S(k, t)$ at intermediate times after the quench, at a larger value of $k$ was observed in the two-dimensional calculations of Mao et al. ${ }^{43}$ where they considered a nonlinear extension of Cahn-Hilliard theory for polymer mixtures. The order parameter for their theory is the deviation of the concentration $c(\mathbf{r}, t)=C(\mathbf{r}, t)-C_{0}$ [i.e., $c(\mathbf{r}, t)$ replaces $\widetilde{\rho}(\mathbf{r}, t)$ in our theory]. They used the approximation that the mobility $M$ $=M_{0}+M_{1} c(\mathbf{r}, t)$, i.e., a linear function of $c(\mathbf{r}, t)$, together with a square-gradient Helmholtz free energy functional in Eq. (31). By linearizing $\delta F / \delta C$ about the average concentration $C_{0}$ in the manner leading to Eq. (34) along with the continuity equation (2), Mao et al. ${ }^{43}$ obtain a nonlinear theory that is very similar in structure to that which we obtain using Eq. (1) with a constant mobility together with the truncated functional (35). The main difference between the two approaches, other than the choice of approximation for the Helmholtz free energy functional, ${ }^{54}$ is that Mao et al. ${ }^{43}$ have an adjustable parameter, the ratio $M_{1} / M_{0}$, that allows a tuning of the mobility for their polymer blend, whereas in our approach we are effectively restricted to the choice $M_{1} / M_{0}=1 / \rho_{b}$. Their results also show that the second peak (shoulder) in $S(k, t)$ results from considering a theory that is nonlinear (second order) in the order parameter. The other significant difference from Ref. 43 is that the present theory uses a microscopic nonlocal functional (35), and therefore includes the effects of interparticle correlations more accurately than a gradient (Ginzburg-Landau) theory such as that used by Mao et al. ${ }^{43}$ Nevertheless, in the early and intermediate times of spinodal decomposition, where sharp interfaces between gaslike and liquidlike domains have not yet formed, we should expect good qualitative agreement between our results and those from the nonlinear CahnHilliard gradient theory of Mao et al. ${ }^{43}$ Mao et al. also found that the presence of the nonlinear terms in their theory resulted in a significant change in the connectivity of contour plots of the order parameter from that found in the absence of these terms $\left(M_{1}=0\right)$ - see Figs. 3 and 5 of Ref. 43. On the basis of our present analysis we would expect this observation to be a general feature of spinodal decomposition at intermediate time scales.

\section{CONCLUDING REMARKS}

This paper falls broadly into two parts. In the first part we provided an alternative derivation of the DDFT developed by MT. ${ }^{3,4}$ Our derivation elucidates the physical approximations made in order to construct the theory. The starting point is the Smoluchowski equation (8), the (generalized) Fokker-Planck equation for the probability distribution function $P\left(\mathbf{r}^{N}, t\right)$ corresponding to the Langevin equation of motion, Eq. (10). This stochastic basis for the theory means that the theory should be applicable to colloidal fluids where because of interactions with the solvent particles the momentum degrees of freedom of the colloids equilibrate much faster than the positional degrees of freedom. In atomic fluids the equilibration time scale for the momentum degrees of freedom can be of the same order as that for the relaxation of positional degrees of freedom and therefore the present theory may break down for atomic fluids. However, because the correct equilibrium limit is built into this theory, i.e., when $\partial \rho(\mathbf{r}, t) / \partial t=0$ the theory is equivalent to Eq. (23), it is feasible that the present theory would give reasonable results for atomic fluids, assuming that one also has a reliable approximation for the excess Helmholtz free energy functional, $F_{\text {ex }}[\rho]$. The fact that the correct equilibrium limit is built in is, we believe, one of the most appealing features of the theory.

The further approximation (beyond assuming that the Smoluchowski equation holds) made in deriving the DDFT, Eq. (18), is to assume Eqs. (16) and (17), exact equilibrium results, are also valid for the nonequilibrium fluid. This approximation is equivalent to assuming that the two-particle, three-particle, and higher order correlations in the nonequilibrium fluid are equivalent to those in an equilibrium fluid with the specified one-body density profile. We expect that this approximation is a reasonable one to make and will not result in significant failures of the theory, as long as other approximations can be justified. We therefore believe that Eq. (18) may well provide a reliable account of the dynamics for a variety of different fluids, provided of course, that one has an accurate approximation for the appropriate $F_{\text {ex }}[\rho]$.

The second part of the present paper is concerned with the application of the DDFT to the problem of spinodal decomposition. Our key result is Eq. (37). For early times after quenching the fluid, when density fluctuations are small in amplitude, our theory reduces to the linear theory of Evans and Telo Da Gama. ${ }^{36}$ Furthermore, this linear theory reduces to the Cahn-Hilliard theory if we were to use a square gradient Helmholtz free energy functional Eq. (32) rather than the more accurate nonlocal functional, Eq. (35). CahnHilliard theory ${ }^{32-35}$ is known to provide a successful qualitative description of the early stages of spinodal decomposition. Since the present theory incorporates all the effects that Cahn-Hilliard theory describes, and provides a more accurate treatment of short wavelength correlations ${ }^{36}$ it should be a reliable quantitative theory for the early stages of spinodal decomposition.

However, the key feature of our theory is that it incorporates a "mode-coupling" term [final term on the righthand side of Eq. (37)] which describes the coupling between different density fluctuation modes, an effect which becomes 
important in the intermediate stages of spinodal decomposition. The results of calculations including our "modecoupling" term are in qualitative agreement with those from a recent nonlinear extension to Cahn-Hilliard theory. ${ }^{43} \mathrm{We}$ believe that further work needs to be done in testing the predictions of our theory. In particular, a comparison with Brownian dynamics simulation results for spinodal decomposition would be very useful; we do not know of relevant simulations which go beyond the very early stages of spinodal decomposition. Of course, running simulations that get into the stage of spinodal decomposition for which our results exhibit deviations from those of the linear theory could be computationally expensive.

We conclude by mentioning one important conceptual issue regarding the input to our theory of spinodal decomposition. Clearly Eqs. (29) or (37) require $\hat{c}(k)$ as input. As emphasized earlier, for an equilibrium state $\hat{c}(k)$ is simply the Fourier transform of the pair direct correlation function and, as such, it can be obtained from integral equation theories ${ }^{31}$ or from simulations. In Ref. 36 results for $\hat{c}(k)$ calculated using Percus-Yevick theory for a Lennard-Jones fluid were extrapolated into the unstable spinodal region. ${ }^{55,56}$ Such a procedure is fraught with uncertainty and is difficult to justify. The present DFT formulation of the theory avoids such problems. $c^{(2)}$ is defined as the second functional derivative of $-\beta F_{\text {ex }}[\rho]$ and provided one makes a division of $F_{\text {ex }}[\rho]$ into a repulsive reference contribution plus an attractive (mean-field-like) contribution, as in Eq. (41), there is no difficulty in calculating $c^{(2)}\left(r ; \rho_{b}\right)$ inside the spinodal. We do not need to make any extrapolation. One could envisage treating attractive interactions in a more sophisticated fashion $^{1}$ than in Eq. (41) but provided the basic division is maintained one should expect to obtain similar results for $\hat{c}(k)$.

\section{ACKNOWLEDGMENTS}

The authors are grateful to Christos Likos, Markus Rauscher, and Jan Dhont for useful discussions and comments on the present work. A.J.A. acknowledges the support of EPSRC under grant No. GR/S28631/01.

${ }^{1}$ For example, R. Evans, in Fundamentals of Inhomogeneous Fluids, edited by D. Henderson (Dekker, New York, 1992), Chap. 3.

${ }^{2}$ S. Dietrich, in Phase Transitions and Critical Phenomena edited by C. Domb and J. L. Lebowitz (Academic, London, 1988), Vol. 12, p. 1.

${ }^{3}$ U. Marini Bettolo Marconi and P. Tarazona, J. Chem. Phys. 110, 8032 (1999).

${ }^{4}$ U. Marini Bettolo Marconi and P. Tarazona, J. Phys.: Condens. Matter 12, A413 (2000).

${ }^{5}$ R. Evans, Adv. Phys. 28, 143 (1979).

${ }^{6}$ W. Dieterich, H. L. Frisch, and A. Majhofer, Z. Phys. B: Condens. Matter 78, 317 (1990)

${ }^{7}$ B. Bagchi, Physica A 145, 273 (1987).

${ }^{8}$ A. Yoshimori, T. J. F. Day, and G. N. Patey, J. Chem. Phys. 108, 6378 (1998).

${ }^{9}$ J. Dzubiella and C. N. Likos, J. Phys.: Condens. Matter 15, L147 (2003).

${ }^{10}$ F. Penna and P. Tarazona, J. Chem. Phys. 119, 1766 (2003).

${ }^{11}$ F. Penna, J. Dzubiella, and P. Tarazona, Phys. Rev. E 68, 061407 (2003).

${ }^{12}$ J. Chakrabarti, J. Dzubiella, and H. Löwen, cond-mat/0403475.

${ }^{13}$ K. Kawasaki, Physica A 208, 35 (1994).
${ }^{14}$ T. R. Kirkpatrick and D. Thirumalai, J. Phys. A 22, L149 (1989).

${ }^{15}$ T. Munakata, J. Phys. Soc. Jpn. 58, 2434 (1989).

${ }^{16}$ T. Munakata, J. Phys. Soc. Jpn. 59, 1299 (1989).

${ }^{17}$ T. Munakata, Phys. Rev. E 50, 2347 (1994).

${ }^{18}$ D. S. Dean, J. Phys. A 29, L613 (1996).

${ }^{19}$ H. Frusawa and R. Hayakawa, J. Phys. A 33, L155 (2000).

${ }^{20}$ K. Kawasaki, J. Phys.: Condens. Matter 12, 6343 (2000).

${ }^{21}$ A. J. Archer and M. Rauscher, cond-mat/0405603.

${ }^{22}$ T. Munakata, Phys. Rev. E 67, 022101 (2003).

${ }^{23}$ P. N. Pusey, in Liquids, Freezing and Glass Transition, edited by J-P. Hansen, D. Levesque, and J. Zinn-Justin (North-Holland, Amsterdam, 1991).

${ }^{24}$ P. N. Pusey and R. J. A. Tough, in Dynamic Light Scattering, edited by R. Pecora (Plenum, New York, 1985).

${ }^{25}$ C. A. Croxton, Liquid State Physics-A Statistical Mechanical Introduction (Cambridge University Press, Cambridge, 1974).

${ }^{26} \mathrm{H}$. Risken, The Fokker-Planck Equation: Methods of Solutions and Applications, 2nd ed. (Springer-Verlag, Berlin, 1996).

${ }^{27}$ C. W. Gardiner, Handbook of Stochastic Methods for Physics, Chemistry and the Natural Sciences (Springer-Verlag, Berlin, 1996).

${ }^{28}$ R. Verberg, I. M. de Schepper, and E. G. D. Cohen, Phys. Rev. E 61, 2967 (2000).

${ }^{29}$ J. K. G. Dhont, J. Chem. Phys. 105, 5112 (1996).

${ }^{30}$ J. K. G. Dhont, A. F. H. Duyndam, and B. J. Ackerson, Langmuir 8, 2907 (1992).

${ }^{31}$ J-P. Hansen and I. R. McDonald, Theory of Simple Liquids, 2nd ed. (Academic, London, 1986).

${ }^{32}$ J. D. Gunton, M. San Miguel, and P. S. Sahni, in Phase Transitions and Critical Phenomena, edited by C. Domb and J. L. Lebowitz (Academic, London, 1988), Vol. 8, p. 267.

${ }^{33}$ A. Onuki, Phase Transition Dynamics (Cambridge University Press, Cambridge, 2002).

${ }^{34}$ J. W. Cahn and J. E. Hilliard, J. Chem. Phys. 31, 688 (1959).

${ }^{35}$ J. W. Cahn, Acta Metall. 9, 795 (1961).

${ }^{36}$ R. Evans and M. M. Telo Da Gama, Mol. Phys. 38, 687 (1979).

${ }^{37}$ F. F. Abraham, J. Chem. Phys. 64, 2660 (1976).

${ }^{38}$ S. M. Allen and J. W. Cahn, Acta Metall. 27, 1085 (1979).

${ }^{39}$ A. J. Bray, Adv. Phys. 43, 357 (1994).

${ }^{40}$ Note that inside the spinodal $S(k)$ should not be interpreted as the static structure factor [one can define a time-dependent structure factor $S(k, t)$, as in Eq. (46)]. Rather $S(k)$ is defined for all state points as (1 $\left.-\rho_{b} \hat{c}(k)\right)^{-1}$, where $\hat{c}(k)$ is defined as the Fourier transform of the second functional derivative of $F_{\text {ex }}[\rho]$, i.e., via (26). Thus $S(k)$ can take negative values inside the spinodal region.

${ }^{41}$ Starting from the square gradient functional (32) it is straightforward to show (Ref. 5) that $1-\rho_{b} \hat{c}(k)=\beta \rho_{b}\left[\left(\partial^{2} f_{0} / \partial \rho^{2}\right)_{\rho_{b}}+K k^{2}\right]$.

${ }^{42}$ We note that there is some similarity between the structure of Eq. (37) and the equations of mode coupling theory-see, for example, E. Zaccarelli, G. Foffi, P. De Gregorio, F. Sciortino, P. Tartaglia, and K. A. Dawson, J. Phys.: Condens. Matter 14, 2413 (2002)—used to describe the glass transition.

${ }^{43}$ Y. Mao, T. C. B. McLeish, P. I. C. Teixeira, and D. J. Read, Eur. Phys. J. E 6, 69 (2001).

${ }^{44}$ See, e.g., C. N. Likos, Phys. Rep. 348, 267 (2001).

${ }^{45}$ S. Asakura and F. Oosawa, J. Chem. Phys. 22, 1255 (1954).

${ }^{46}$ S. Asakura and F. Oosawa, J. Polym. Sci. 33, 183 (1958).

${ }^{47}$ For a recent summary see J. M. Brader, R. Evans, and M. Schmidt, Mol. Phys. 101, 3349 (2003).

${ }^{48}$ Y. Rosenfeld, Phys. Rev. Lett. 63, 980 (1989).

${ }^{49}$ Y. Rosenfeld, D. Levesque, and J.-J. Weis, J. Chem. Phys. 92, 6818 (1990).

${ }^{50}$ Y. Rosenfeld, J. Chem. Phys. 93, 4305 (1990).

${ }^{51}$ N. W. Ashcroft and J. Lekner, Phys. Rev. 145, 83 (1966).

${ }^{52}$ F. F. Abraham, M. R. Mruzic, and G. M. Pound, J. Chem. Phys. 70, 2577 (1979).

${ }^{53}$ J. F. M. Lodge and D. M. Heyes, J. Chem. Phys. 109, 7567 (1998).

${ }^{54}$ In Ref. 43 the Flory-Huggins-de Gennes form is used for $F[C(\mathbf{r}, t)]$.

${ }^{55}$ C. Ebner, W. F. Saam, and D. Stroud, Phys. Rev. A 14, 2264 (1976).

${ }^{56}$ M. M. Telo Da Gama and R. Evans, Mol. Phys. 38, 367 (1979). 